TITLE:

\title{
Mode-selective phonon excitation in gallium nitride using mid- infrared free-electron laser
}

\section{AUTHOR(S):}

Kagaya, Muneyuki; Yoshida, Kyohei; Zen, Heishun; Hachiya, Kan; Sagawa, Takashi; Ohgaki, Hideaki

\section{CITATION:}

Kagaya, Muneyuki ...[et al]. Mode-selective phonon excitation in gallium nitride using midinfrared free-electron laser. Japanese Journal of Applied Physics 2017, 56(2): 0200701.

\section{ISSUE DATE:}

2017-01-13

URL:

http://hdl.handle.net/2433/245811

\section{RIGHT:}

(c) 2017 The Japan Society of Applied Physics; This is an author-created, un-copyedited version of an article accepted for publication in 'Japanese Journal of Applied Physics'. The publisher is not responsible for any errors or omissions in this version of the manuscript or any version derived from it. The Version of Record is available online at https://doi.org/10.7567/J JAP.56.022701; The full-text file will be made open to the public on 13 January 2018 in accordance with publisher's 'Terms and Conditions for Self-Archiving': この論文は出版社版でありません。引用の際に は出版社版をご確認ご利用ください。; This is not the published version. Please cite only the published version. 


\section{Mode-selective phonon excitation in gallium nitride using mid-infrared free-electron laser}

Muneyuki Kagaya, Kyohei Yoshida ${ }^{1}$, Heishun Zen², Kan Hachiya*, Takashi Sagawa, and Hideaki Ohgaki

Department of Fundamental Energy Science, Graduate School of Energy Science, Kyoto University, Kyoto 606-8501, Japan

${ }^{1}$ Department of Applied Chemistry and Biochemistry, Graduate School of Science and Technology, Kumamoto University, Kumamoto 860-8555, Japan

${ }^{2}$ Advanced Energy Generation Division, Institute of Advanced Energy, Kyoto University, Uji, Kyoto 611-0011, Japan

The single-phonon mode was selectively excited in a solid-state sample. A mid-infrared free-electron laser, which was tuned to the target phonon mode, was irradiated onto a crystal cooled to a cryogenic temperature, where modes other than the intended excitation were suppressed. An $A_{1}(\mathrm{LO})$ vibrational mode excitation on $\mathrm{GaN}(0001)$ face was demonstrated. Anti-Stokes Raman scattering was used to observe the excited vibrational mode, and the appearance and disappearance of the scattering band at the target wavenumber were confirmed to correspond to on and off switching of the pump free-electron laser and were fixed to the sample vibrational mode. The sum-frequency generation signals of the pump and probe lasers overlapped the Raman signals and followed the wavenumber shift of the pump laser.

\section{Introduction}

Not only thermal and structural properties, but also a number of electronic properties are directly connected to the phonon mode, in normal conductivity and superconductivity as well as in quantum dynamics. ${ }^{1-3)}$ By exciting a particular phonon mode, electron-phonon interactions even induce metal-insulator transitions ${ }^{3)}$ and changes in the magnetic order, ${ }^{4)}$ for example. The role of an individual mode with respect to these physical properties has been investigated for both acoustic ${ }^{5-7)}$ and optical phonons. ${ }^{8,9)}$ The coherent phonon spectroscopy technique, ${ }^{10,11)}$ in which a stimulated Raman effect is utilized, is the most typical method of exciting the target phonon mode. ${ }^{12-15)}$ It has been successfully applied to clarify the relationship between each mode and the emergence of the above physical properties. ${ }^{2-9)}$ This method utilizes laser pulses shorter

\footnotetext{
*E-mail: hachiya@energy.kyoto-u.ac.jp
} 
than the period of lattice vibration, and the oscillation spectrum bandwidth of the laser pulses is, in principle, wider than the frequency of the phonon to be selectively excited. In order to realize both successful excitation and selectivity of the single mode, not only the narrowness of the pulse time width, which leads to a wide spectrum ensuring phonon excitation, but also subtle control of the pulse shape, which excludes excitations of the additional phonon modes, is necessary. One of the above applications is $E_{1}$-mode excitation in GaN by a sophisticated femtosecond pulse shaping technique. ${ }^{16)}$

In a previous report, some of the present authors proposed an alternative method with a more simple and straightforward approach, involving the direct pumping of the phonon energy through the irradiation of mid-infrared (MIR) free-electron-laser (FEL) light whose energy is equal to that of the target phonon. ${ }^{17)}$ Selective excitation of the $\mathrm{FLO}(0)$ phonon mode of $A_{1}$-type vibration, which is at $970 \mathrm{~cm}^{-1}$ in $6 \mathrm{H}-\mathrm{SiC}$, was demonstrated. In this method, it is possible to easily confine the bandwidth of the pump laser wavelength within a limited range around that of the individual target phonon mode, compared with the coherent phonon spectroscopy technique. The selectivity around the target frequency is retained by the micropulse duration, which is $0.6 \mathrm{ps},{ }^{17}$ ) and the lifetime of the excited states created by the micropulse, which is $3-10 \mathrm{ps}^{18,19)}$ This property is expected to stabilize the excited vibrational state against mode transition. ${ }^{2,20)}$

In the present paper, we report an application of our method to one of the most typical wide-gap semiconductors, GaN, which is of the wurtzite structure and has long been studied. ${ }^{21-24)}$ According to the results of infrared (IR) and Raman spectroscopy, GaN has both $\mathrm{IR}^{25,26)}$ and Raman active modes, ${ }^{27-29)}$ which can be excited by one photon and observed by common anti-Stokes (AS) Raman spectroscopy, ${ }^{17)}$ within the present wavenumber range of the oscillating frequency of the FEL we use (Kyoto University Free-Electron Laser: KU-FEL $\left.{ }^{30)}\right)$ of approximately $450-2,000 \mathrm{~cm}^{-1}(5-22 \mu \mathrm{m}) .{ }^{31)}$

\section{Experimental methods}

MIR-FEL was irradiated as a pumping light source to excite the phonon mode, and a $532 \mathrm{~nm}$ laser was selected for the probe in the AS Raman scattering measurement. The experimental setup for the optical irradiation and measurement system is shown in Fig. 1. The second-harmonic wave of the Nd:YAG laser (Continuum Surelite SLII-10) was used as a probe. The full width at half maximum (FWHM) of the pulse duration is 4-6 ns for the probe laser. As explained in the previous report, ${ }^{17)}$ the pump laser, KU- 
FEL, has a specific pulse structure with two types of pulses: macro- and micropulses with durations of $2 \mu \mathrm{s}$ (FWHM) and $0.6 \mathrm{ps}$ (FWHM) at $12 \mu \mathrm{m}$, respectively. ${ }^{31,32)}$ The reported lifetimes of the phonons in GaN exceed $2 \mathrm{ps},{ }^{19)}$ and the pulse width of the micropulse of KU-FEL is shorter than the phonon relaxation time. Therefore, the MIRFEL irradiation induces mode-selective phonon excitation via a photoexcitation effect. The long macropulse, in contrast, enables the repetitive excitation and observation of the target phonon mode under an effectively constant condition. Synchronization between pump and probe lasers was accomplished using function and delay generators, and it was confirmed on an oscilloscope.

The (0001) face of single-crystal GaN (Sinyo) with a wurtzite structure was used for the measurement. All the Raman scattering measurements were conducted with backscattering configuration against the $c$-plane. $E_{2}$ and $A_{1}(\mathrm{LO})$ are the phonon modes observed in this configuration. ${ }^{29)}$ The sample was cooled to $13 \mathrm{~K}$ in a cryostat (Taiyo Nippon Sanso 1005 337) equipped with a helium compressor (Sumitomo Heavy Industries CKW-21A). As noted in the figure, a $\mathrm{KBr}$ window was set to pass both the MIR pump and the $532 \mathrm{~nm}$ probe laser light, and a fused quartz window was mounted to transmit the scattered light.

The scattered light was guided through triply stacked notch filters (Thorlabs NF533$17 \times 2$; Opto-line StopLine notch filter NF03-532E-25 ×1) in order to eliminate Rayleigh scattering light of the probe laser, and was detected by photon counting with a photomultiplier (Hamamatsu Photonics R3896 and C1392) after dispersion with a spectrometer (Horiba Scientific Triax 190).

\section{Results and discussion}

The $A_{1}(\mathrm{LO})$ mode at around $\left.734 \mathrm{~cm}^{-1} 28,29\right)$ was chosen as the target. As clarified in the previous study, ${ }^{17}$ ) we chose this phonon mode because it is both Raman and IR active. Before the phonon excitation measurement, we confirmed the $A_{1}(\mathrm{LO})$ mode (Stokes) Raman signal of the present sample in a preliminary measurement. The spectrum was obtained with a spectrometer (Ocean Optics QEPro-Raman) with a Raman probe (Ocean Optics RIP-RPB-785), and a laser of $785 \mathrm{~nm}$ wavelength (Ocean Optics Laser-785) was used for excitation. As presented in Fig. 2, the Stokes Raman signal was observed at $731.5 \mathrm{~nm}$ at room temperature. The FEL wavelength was tuned to $13.67 \mu \mathrm{m}$, which corresponds to $731.5 \mathrm{~cm}^{-1}$. Figure 3 shows the AS Raman scattering measurement results over a wide range of wavenumber with and without FEL irradia- 
tion, whose spectrum center is tuned to $731.5 \mathrm{~cm}^{-1}$. The pump-FEL spectrum is also given in the upper panel of Fig. 3. The FEL macropulse energy was set to 3.8 mJ. This corresponds to $\sim 100 \mathrm{~mJ} \mathrm{~cm}^{-2}$ in the present measurement system. ${ }^{33)}$ The spectrometer slit width was $0.3 \mathrm{~mm}$. The spectra were obtained using the 300-times average of each point. The pulse energy of the Nd:YAG probe laser was $10.2 \mu \mathrm{J}$. The AS Raman band at around $734 \mathrm{~cm}^{-1}$, assigned to $A_{1}(\mathrm{LO})$, followed on/off switching of the pump FEL, while another AS band at $565 \mathrm{~cm}^{-1}$, assigned to $E_{2}^{\mathrm{H}}$ (the emergence of this phonon mode in wurtzite GaN is clearly explained in Ref. 29), did not exhibit any significant difference with or without FEL irradiation. It should also be noted that cooling of the sample did not totally suppress the AS Raman signal, especially for $E_{2}^{\mathrm{H}}$, which is originally a phonon mode of an intensive Raman scattering cross section, as indicated in Fig. 2. The residual signal possibly originated from the intense probe laser, which may have stimulated the Stokes Raman scattering process to generate a non-negligible number of phonons, for example.

As explained in the experimental section and discussed thoroughly in the previous report, ${ }^{17)}$ our mid-IR FEL excites phonons of a $3-5$ ps lifetime ${ }^{19)}$ by irradiating $0.6 \mathrm{ps}$ micropulses, and the probe nanosecond Nd:YAG laser light is modulated to contain an AS Raman signal with the repeatedly excited phonons generated by the micropulses in a microsecond macropulse. Discrete micropulses with 350 ps intervals (Fig. 2 in Ref. 17) are expected to create a one-phonon-excited state with one photon repeatedly over a $2 \mu$ s pulse length of a macropulse if we choose an appropriate excitation photon density. With the present pump-and-probe laser combination under the experimental conditions, the sum-frequency generation (SFG) signal overlapped the AS Raman signal. This was confirmed through the irradiation of the FEL with wavenumbers shifted from $A_{1}(\mathrm{LO})$ as well as the wavenumber tuned to the target phonon mode. The AS Raman and SFG spectra are presented in Fig. 4 with pump FEL spectra. The wavelengths of the FEL were $13.67,12.70$, and $12.25 \mu \mathrm{m}$, which corresponded to $731.5,787.4$, and $816.3 \mathrm{~cm}^{-1}$, respectively. The macropulse energies were set to $6.0,8.0$, and $8.8 \mathrm{~mJ}$, respectively. The Nd:YAG pulse energy was $11.3 \mu \mathrm{J}$. The spectrometer slit width was narrowed to $0.2 \mathrm{~mm}$, while the displayed spectra were obtained using 700-times averages of each point in order to obtain AS Raman spectra at close intervals. In both spectra with the wavenumber-shifted FEL of 787.4 and $816.3 \mathrm{~cm}^{-1}$, signals of moderate intensities were observed at the wavenumbers corresponding to those of the pump FEL. The spectrum for $787.4 \mathrm{~cm}^{-1}$ seems to contain another signal from 720 to $750 \mathrm{~cm}^{-1}$, centered at around 
$730 \mathrm{~cm}^{-1}$. This additional weak band arises because the FEL spectrum extends its tail to the $A_{1}(\mathrm{LO})$-signal position, as presented in the upper panel of Fig. 4. It is suggested that the irradiation of the FEL and Nd:YAG laser under the present conditions yields both AS Raman and SFG signals. The SFG signal followed the shift of the FEL wavenumber, while the AS Raman signal induced by phonon excitation did not because the phonon energy is constant. Within this interpretation, the difference in the bandwidth between the narrow fixed AS Raman band at around $730 \mathrm{~cm}^{-1}$ and the broad floating SFG band that follows $784 \mathrm{~cm}^{-1}$ FEL irradiation is due to the difference in the spectra between the sharp AS Raman band from a single crystal of high crystallinity and the relatively extended structure of the line-shape function for the FEL oscillation frequency. This is why the narrow band is added at $730 \mathrm{~cm}^{-1}$ even when the FEL intensity is low. The overlap of the sum-frequency generation signal on the AS Raman scattering band of excited phonons outside the reststrahlen band ${ }^{25)}$ was also observed in $6 \mathrm{H}-\mathrm{SiC}$ when a picosecond laser ${ }^{18,34)}$ was used as a probe instead of a nanosecond laser. ${ }^{17)}$

Another possible explanation for the overlap is that the spectrum structure arising from the resonant frequency of nonlinear susceptibility within the SFG process shows a distinct narrow band. ${ }^{35)}$ A similar structure was observed by second-harmonicgeneration spectroscopy for $4 \mathrm{H}-\mathrm{SiC}$, although the effect of the Fresnel transmission tensor was also taken into account. ${ }^{36)}$ The structure could be clearly observed again in the present measurement when the optical paths were carefully aligned and arranged to fulfill the phase-matching condition. ${ }^{37)}$ As illustrated in the inset in Fig. 1, the measurement of the scattered light was performed in the direction close to the normal vector of the sample face. In our case, the direction of measurement was at a large angle to the SFG wave vector of phase matching. This means that the SFG intensity was expected to be low because of the failure of the phase-matching condition.

\section{Conclusions}

Mid-infrared free-electron-laser light was tuned to the wavenumber of the $A_{1}(\mathrm{LO})$ vibrational mode for single-phonon excitation at the $\mathrm{GaN}(0001)$ face at a low temperature. We confirmed successful phonon excitation through anti-Stokes Raman scattering. We also confirmed the overlap of the sum-frequency generation signal of pump and probe lasers on the anti-Stokes Raman scattering signal of the excited phonon, although only the Raman signal followed the on/off switching of the pump free-electron laser. 


\section{Acknowledgment}

This work was supported by JSPS KAKENHI Grant Number 26420884 and the "Joint Usage/Research Program on Zero-Emission Energy Research", Institute of Advanced Energy, Kyoto University (ZE26A-26 and ZE27A-7). 


\section{References}

1) W. S. Warren, H. Rabitz, and M. Dahleh, Science 259, 1581 (1993).

2) M. Först, C. Manzoni, S. Kaiser, Y. Tomioka, Y. Tokura, R. Merlin, and A. Cavalleri, Nat. Phys. 7, 854 (2011).

3) M. Rini, R. Tobey, N. Dean, J. Itatani, Y. Tomioka, Y. Tokura, R. W. Schoenlein, and A. Cavalleri, Nature 449, 72 (2007).

4) M. Först, R. I. Tobey, S. Wall, H. Bromberger, V. Khanna, A. L. Cavalieri, Y.-D. Chuang, W. S. Lee, R. Moore, W. F. Schlotter, J. J. Turner, O. Krupin, M. Trigo, H. Zheng, J. F. Mitchell, S. S. Dhesi, J. P. Hill, and A. Cavalleri, Phys. Rev. B 84, 241104(R) (2011).

5) D. H. Hurley, R. Leis, O. B. Wright, and O. Matsuda, Appl. Phys. Lett. 93, 113101 (2008).

6) A. Bartels, T. Dekorsy, H. Kurz, and K. Köhler, Appl. Phys. Lett. 72, 2844 (1998).

7) C.-K. Sun, Y.-K. Huang, J.-C. Liang, A. Abare, and S. P. DenBaars, Appl. Phys. Lett. 78, 1201 (2001).

8) M. Hase, K. Mizoguchi, H. Harima, S. Nakashima, M. Tani, K. Sakai, and M. Hangyo, Appl. Phys. Lett. 69, 2474 (1996).

9) Y. Kasai, D. Suzuki, H. Kunugita, and K. Ema, J. Lumin. 129, 1820 (2009).

10) W. A. Kütt, W. Albrecht, and H. Kurz, IEEE J. Quantum Electron. 28, 2434 (1992).

11) R. Merlin, Solid State Commun. 102, 207 (1997).

12) D. M. Riffe and A. J. Sabbah, Phys. Rev. B 76, 085207 (2007).

13) T. E. Stevens, J. Kuhl, and R. Merlin, Phys. Rev. B 65, 144304 (2002).

14) L. Dhar, J. A. Rogers, and K. A. Nelson, Chem. Rev. 94, 157 (1994).

15) Y.-X. Yan, E. B. Gamble Jr., and K. A. Nelson, J. Chem. Phys. 83, 5391 (1985).

16) K. G. Lee, D. S. Kim, K. J. Yee, and H. S. Lee, Phys. Rev. B 74, 113201 (2006).

17) K. Yoshida, T. Sonobe, H. Zen, K. Hachiya, K. Okumura, K. Mishima, M. Inukai, H. Negm, T. Konstantin, M. Omer, T. Kii, K. Masuda, and H. Ohgaki, Appl. Phys. Lett., 103, 182103 (2013).

18) T. Murata, T. Katsurayama, T. Kii, T. Konstantin, K. Masuda, T. Nogi, H. Ohgaki, S. Suphakul, H. Zen, K. Yoshida, and K. Hachiya, Proc. FEL2015, 2015, p. 615 .

19) K. T. Tsen, D. K. Ferry, A. Botchkarev, B. Sverdlov, A. Salvador, and H. Morkoc, 
Appl. Phys. Lett. 72, 2132 (1998).

20) T. P. Martin and L. Genzel, Phys. Status Solidi B 61, 493 (1974).

21) J. I. Pankove, J. Lumin. 7, 114 (1973).

22) I. Akasaki, Rev. Mod. Phys. 87, 1119 (2015).

23) H. Amano, Rev. Mod. Phys. 87, 1133 (2015).

24) S. Nakamura, Rev. Mod. Phys. 87, 1139 (2015).

25) A. S. Barker, Jr. and M. Ilegems, Phys. Rev. B 7, 743 (1973).

26) H. Sobotta, H. Neumann, R. Franzheld, and W. Seifeld, Phys. Status solidi B 174, K57 (1992).

27) V. Yu. Davydov, N. S. Averkiev, I. N. Goncharuk, D. K. Nelson, I. P. Nikitina, A. S. Polkovnikov, A. N. Smirnov, and M. A. Jacobson, J. Appl. Phys. 82, 5097 (1997).

28) V. Yu. Davydov, Yu. E. Kitaev, I. N. Goncharuk, A. N. Smirnov, J. Graul,

O. Semchinova, D. Uffmann, M. B. Smirnov, A. P. Mirgorodsky, and

R. A. Evarestov, Phys. Rev. B 58, 12899 (1998).

29) H. Harima, J. Phys.: Condens. Matter 14, R967 (2002).

30) H. Ohgaki, T. Kii, K. Masuda, H. Zen, S. Sasaki, T. Shiiyama, R. Kinjo, K. Yoshikawa, and T. Yamazaki, Jpn. J. Appl. Phys. 47, 8091 (2008).

31) H. Zen, M. Inukai, K. Okumura, K. Mishima, K. Torgasin, H. Negm, M. Omer, K. Yoshida, R. Kinjo, T. Kii, K. Masuda, and H. Ohgaki, Proc. FEL2013, 2013, p. 711 .

32) Y. Qin, H. Zen, X. Wang, T. Kii, T. Nakajima, and H. Ohgaki, Opt. Lett. 38, 1068 (2013).

33) T. Murata, Master's Thesis, Graduate School of Energy Science, Kyoto University, Kyoto (2016).

34) T. Katsurayama, T. Murata, K. Yoshida, H. Zen, K. Hachiya, T. Nogi, S. Suphakul, T. Konstantin, T. Kii, K. Masuda, and H. Ohgaki, to be presented at 41st IRMMW-THz, 2016.

35) R. W. Boyd, Nonlinear Optics (Academic Press, San Diego, CA, 2003) 2nd ed., Chap. 2.

36) A. Paarman, I. Razdolski, A. Melnikov, S. Gewinner, W. Schöllkopf, and M. Wolf, Appl. Phys. Lett. 107, 081101 (2015).

37) Y. R. Shen, The Principles of Nonlinear Optics (Wiley, New York, 1984) Chap. 6. 


\section{List of figures}

Fig. 1. (Color online) Schematic for the MIR-FEL irradiation and anti-Stokes Raman scattering measurement. The inset in the circle shows the geometry around the sample in the cryostat and indicates the spatial configuration of the pump MIR-FEL and probe Nd:YAG laser.

Fig. 2. (Color online) Spectrum obtained by Stokes Raman scattering measurement of GaN(0001) at room temperature.

Fig. 3. (Color online) FEL spectrum of pump laser light irradiated on the sample (upper panel) and spectra obtained by anti-Stokes Raman scattering measurements with and without FEL irradiation (lower panel).

Fig. 4. (Color online) FEL spectra tuned to or shifted from $A_{1}(\mathrm{LO})$-mode wavenumber (upper panel) and spectra obtained by anti-Stokes Raman scattering measurements with and without FEL irradiation (lower panel). 


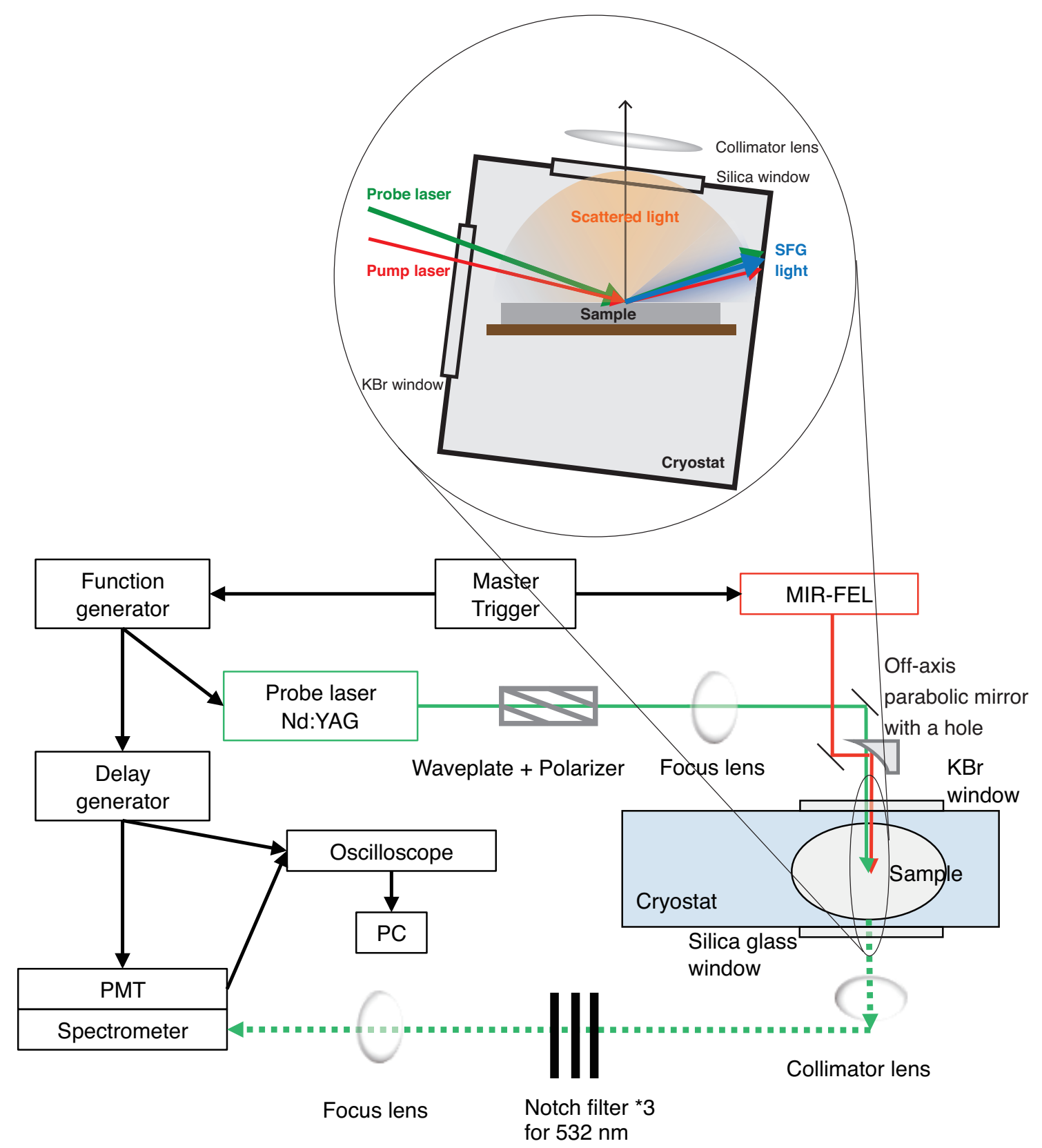

Fig. 1. (Color online) Schematic for the MIR-FEL irradiation and anti-Stokes Raman scattering measurement. The inset in the circle shows the geometry around the sample in the cryostat and indicates the spatial configuration of the pump MIR-FEL and probe Nd:YAG laser. 


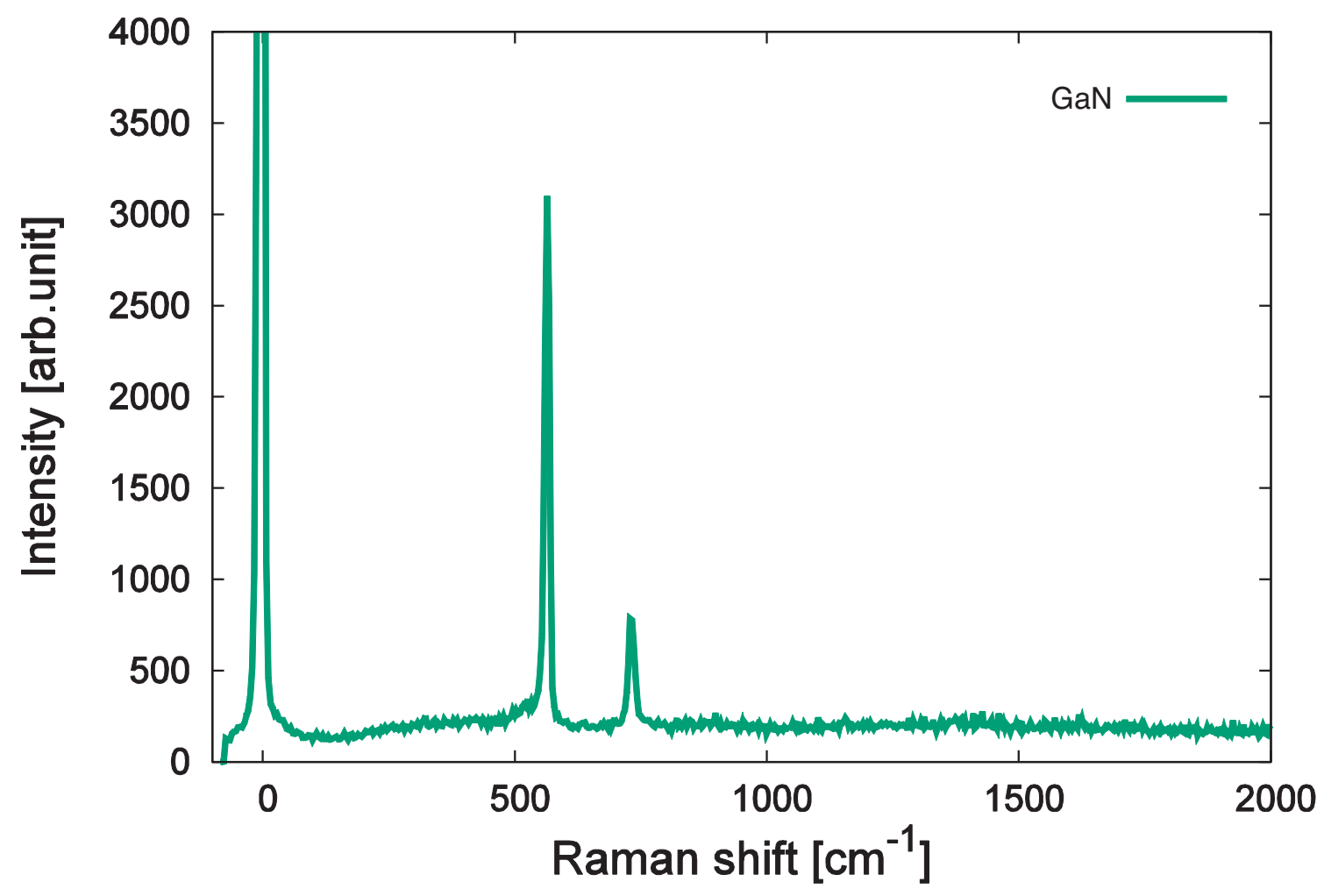

Fig. 2. (Color online) Spectrum obtained by Stokes Raman scattering measurement of $\mathrm{GaN}(0001)$ at room temperature. 

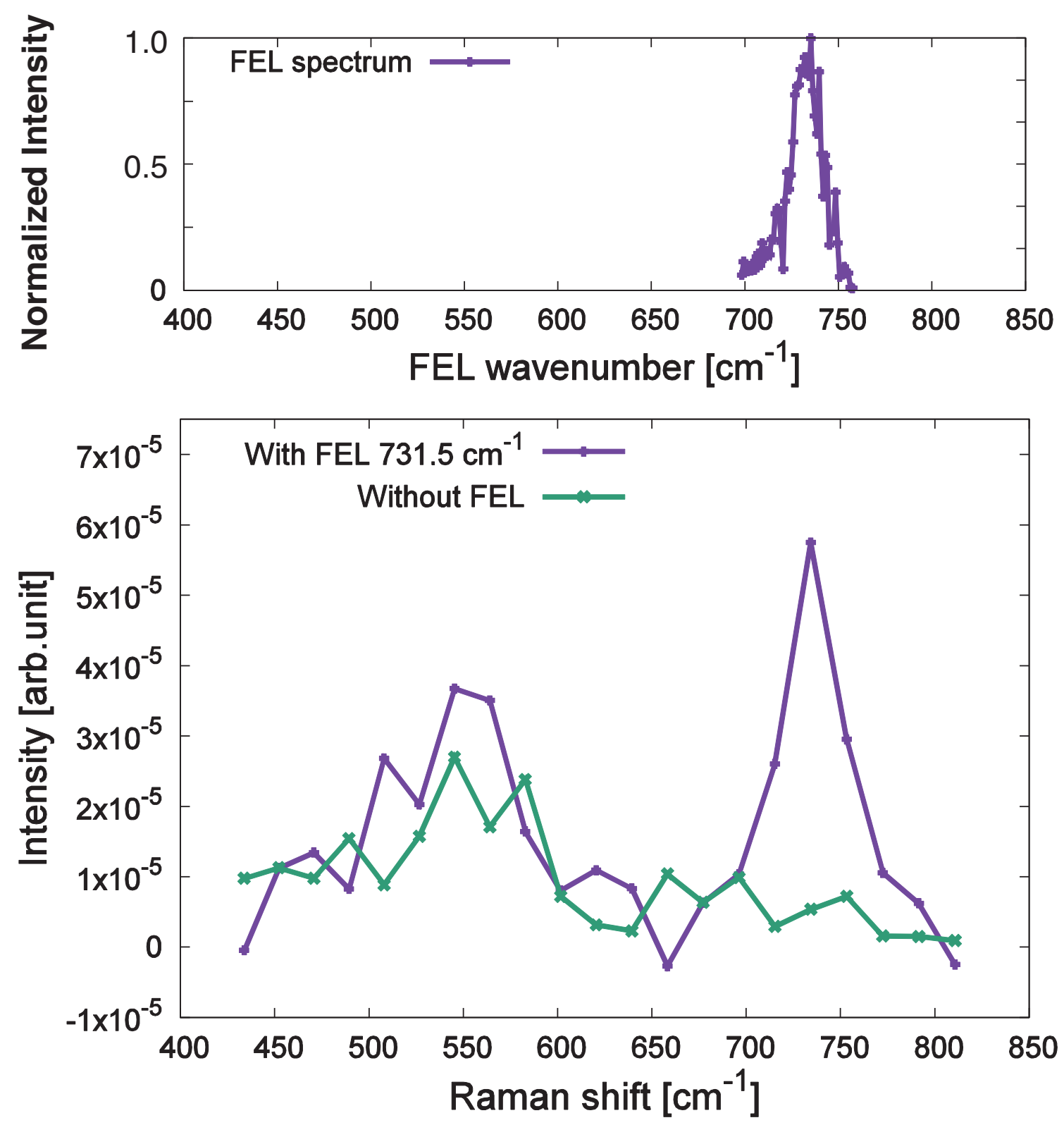

Fig. 3. (Color online) FEL spectrum of pump laser light irradiated on the sample (upper panel) and spectra obtained by anti-Stokes Raman scattering measurements with and without FEL irradiation (lower panel). 

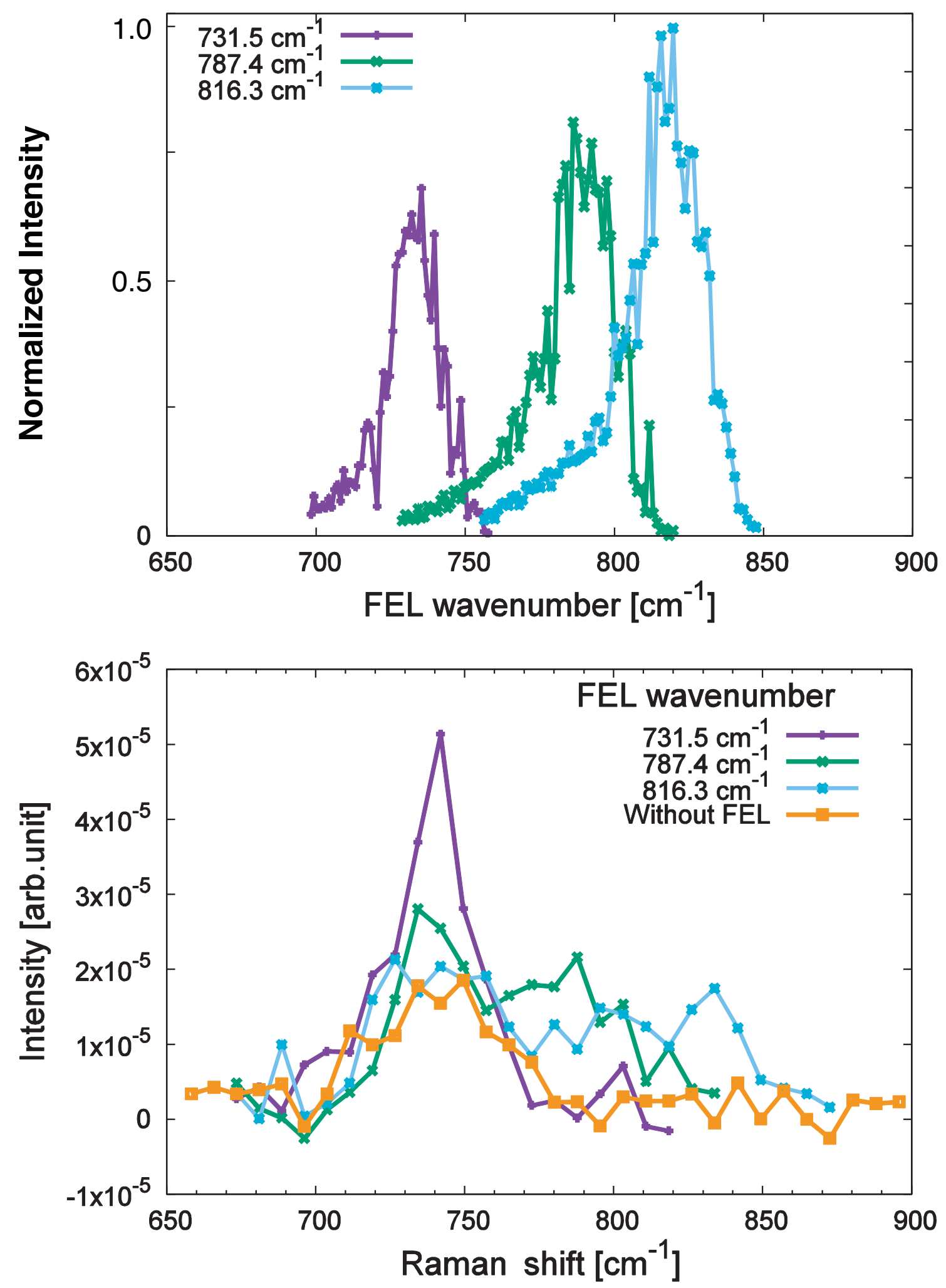

Fig. 4. FEL spectra tuned to or shifted from $A_{1}(\mathrm{LO})$-mode wavenumber (upper panel) and spectra obtained by anti-Stokes Raman scattering measurements with and without FEL irradiation (lower panel). 